\title{
Thrombin generation test in microfluidic systems
}

\author{
Kaspar Koch, Sander S. van Berkel, Marloes M. E. B. van de Wal, Pieter J. Nieuwland, \\ Jan C. M. van Hest, ${ }^{\text {a) }}$ and Floris P. J. T. Rutjes ${ }^{\text {a) }}$ \\ Institute for Molecules and Materials, Radboud University Nijmegen, Toernooiveld 1, 6525 ED Nijmegen, \\ The Netherlands
}

(Received 30 October 2007; accepted 9 December 2007; published online 19 May 2009)

\begin{abstract}
The thrombin generation test is one of the diagnostic tests currently in use as a universal method for measuring hemostatic disorders. We envisioned that conventional monitoring of thrombin generation could be miniaturized resulting in a time-saving, accurate, easy-to-operate, and cost-efficient test. For the translation of the conventional thrombin generation test to microfluidic devices, our focus was directed to parameters such as the detection limit, temperature, protein-surface interactions (i.e., hydrophilicity of microchannels), and mixing behavior. Scaling down to microchannels (e.g., capillaries) resulted in volume reduction and allowed us to study the effect of a microchannel surface (either hydrophilic or hydrophobic) on the thrombin activity. Finally, the use of a micromixer enabled us to perform efficient on-chip mixing, resulting in the successful measurement of a thrombin generation in a microfluidic device.
\end{abstract}

(c) 2009 American Institute of Physics. [DOI: 10.1063/1.3116634]

\section{INTRODUCTION}

Coronary heart disease, stroke and other cerebrovascular diseases, chronic obstructive pulmonary disease, HIV/AIDS, and lower respiratory infections are currently the primary causes of death worldwide. ${ }^{1}$ With more than $22 \times 10^{6}$ fatalities annually, the demand for cheap, rapid, and reliable diagnostic tests for these diseases is ever increasing. ${ }^{2}$ In particular, cardiac diagnostic tests have received significant attention as cardiovascular diseases cover nearly $60 \%$ of all mortalities. One of the diagnostic tests currently in use as a universal method for measuring hemostatic disorders is the thrombin generation test (TGT) developed by Giesen and co-workers. ${ }^{3-5}$ This hemostatic disorders diagnostic tool, however, involves a highly complex, costly, and timeconsuming procedure that needs to be performed by a laboratory specialist. In contrast to conventional diagnostics, novel technology enabled by developments in microfluidics allows rapid diagnosis of blood or plasma samples at the patient's bed, the so-called point-of-care tests. ${ }^{6,7}$ Ideally, such tests can even be carried out by a patient himself enabling rapid diagnosis, which then facilitates the patient to adjust his medical treatment and monitor the development of his disease.

Since the introduction of microfluidic systems by Manz et al. ${ }^{8}$ miniaturization of many chemical and biological processes has experienced a rapid growth. ${ }^{9}$ As evidenced by the number of commercial applications described, application of microfluidic systems in a clinical environment has increased drastically. ${ }^{10}$ Several restrictions such as the detection limit of the analytes, signal to noise ratio in the system, and/or photobleaching of the fluorescent samples, ${ }^{11}$ however, still limit the number of diagnostic applications. Nevertheless, in the field of microfluidics a great interest exists for cardiac

\footnotetext{
${ }^{a)}$ Authors to whom correspondence should be addressed. Electronic addresses: j.vanhest@science.ru.nl and f.rutjes@science.ru.nl.
}

diagnostics. Schembri et al. ${ }^{12}$ were the first to describe a whole blood multiple analyte analyzer using centrifugation and capillary forces. This centrifugal process for the extraction of plasma from sediment was later also used by Haeberle et al. ${ }^{13}$ Other separation techniques applied were based on the bifurcation law ${ }^{14}$ or interchannel microstructures. ${ }^{15}$ Work by Song et al. ${ }^{16}$ demonstrated that clotting times of whole blood and plasma could be determined in very small volumes using a plug-based microfluidic system. Elaborating on the results of Hemker et al., ${ }^{5}$ we envisioned that conventional monitoring of thrombin generation could also be miniaturized resulting in a time-saving, accurate, easy-tooperate, and cost-efficient test. More importantly, lab-on-achip technology enables the use of a diversity of reagents for simultaneous determination of different clotting factors integrating multiple diagnostic tests onto one microchip. Automation of the whole sequence of handling will result in a more robust and reliable system that is less error prone as compared to the conventional test. In this contribution we report the first example of a miniaturized thrombin generation test on a chip.

\section{EXPERIMENTAL DETAILS}

\section{A. Equipment}

Fluorescence microscopy was performed on a Zeiss Axiovert $135 \mathrm{TV}$ with a $10 \times / 0.50$ objective equipped with a DAPI-Aniline blue filter, $\lambda_{\mathrm{ex}} 385 \mathrm{~nm}$. Images were recorded by a Photometrics Coolsnap Camera with a shutter speed of 12 s. As a 96-wells plate fluorometer a Multicounter Wallac Victor2 (equipped with an Umbelliferone filter), $\lambda_{\mathrm{ex}} 385 \mathrm{~nm}$, $T=25{ }^{\circ} \mathrm{C}$ was used. The setup that was used to perform the TGT in a microreactor is schematically depicted in Fig. 1(a). A commercially available microreactor (type TD18, internal volume $3.8 \mu \mathrm{l}$ ) was purchased from Micronit Microfluidics BV (Enschede, The Netherlands). All syringes (Harvard apparatus; high pressure syringe, $2.5 \mathrm{ml}$ ) mounted on a syringe 

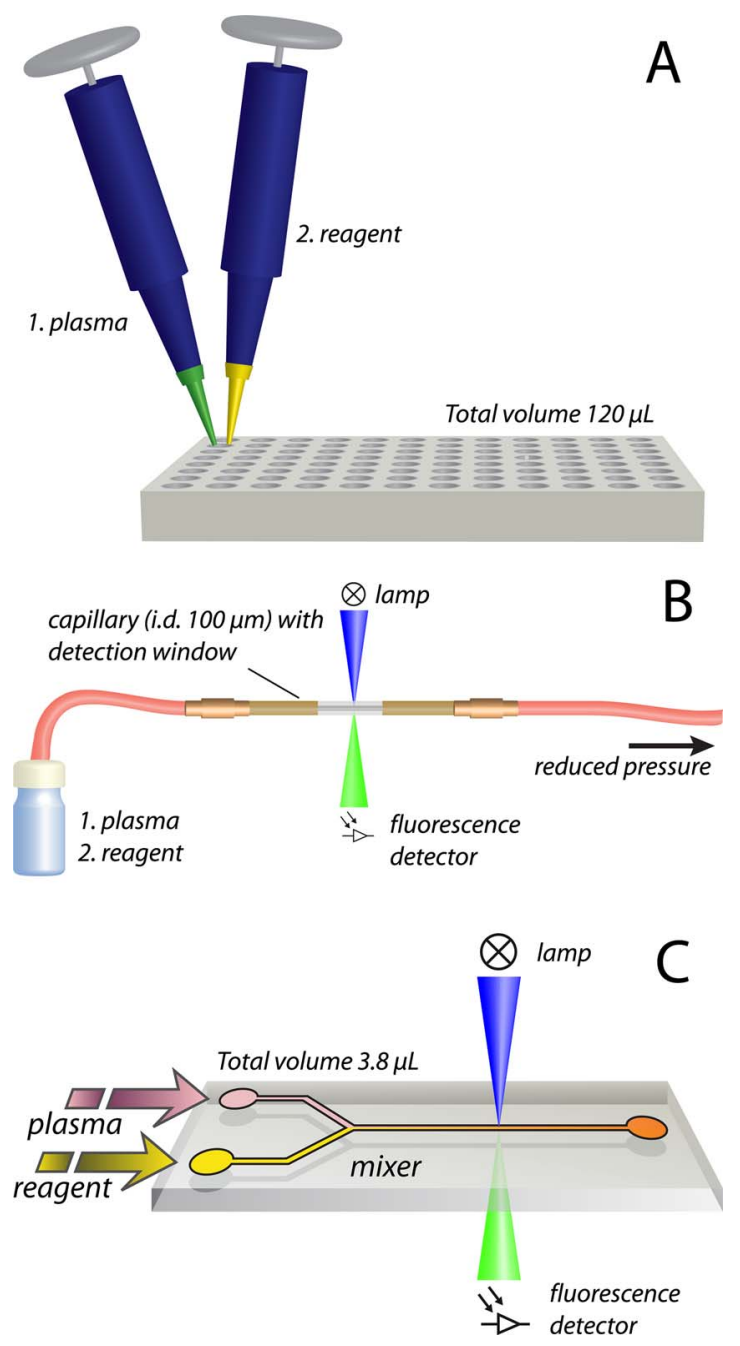

FIG. 1. (Color online) (a) Schematic representation of conventional TG testing. (b) Schematic representation of the setup for TG-Testing in capillaries. (c) Schematic representation of the microreactor setup used for the TGT experiments.

pump (New Era; type NE-1000) were connected using Swagelok connections to FEP tubing (Upchurch Scientific; type: 1529L 1/16" OD, 1/100" ID). At the end of each tubing, a special flat "bottom headless nut" (Upchurch Scientific; type: M-660) was introduced, which pressed down onto a special flat bottom ferrule (Upchurch Scientific; type: M-650) to achieve a gas tight fluid connection to the microreactor. The microreactor was positioned in a chipholder (custom built $)^{17}$ with threaded holes on the top side in which the nuts were screwed. The chipholder was positioned onto the fluorescence microscope during the measurements.

\section{B. Materials}

Fluorogenic substrate Cbz-Gly-Gly-Arg-AMC (I-1140) was obtained from Bachem A.G. (Bubendorf, Switzerland). A solution referred to as Fluo contained $7.5 \mu \mathrm{l}$ of a $100 \mathrm{mM}$ solution of Cbz-Gly-Gly-Arg-AMC in dimethylsulfoxide (DMSO) and $292.5 \mu \mathrm{l}$ buffer (Hepes $20 \mathrm{mM}, p H$ 7.35, containing 60 g/l BSA (bovine serum albumin, Sigma, A-7030), referred to as BSA60). A solution referred to as FluCa contained $25 \mu \mathrm{l}$ of a $100 \mathrm{mM}$ solution of Cbz-Gly-Gly-Arg-
AMC in DMSO, $875 \mu$ l buffer (BSA60) and $100 \mu \mathrm{l} 1 M$ $\mathrm{CaCl}_{2}$, resulting in a concentration of $2.5 \mathrm{mM}$ in fluorogenic substrate and $100 \mathrm{mM}$ in $\mathrm{CaCl}_{2}$. Platelet poor plasma (PPP) was prepared according to literature ${ }^{5}$ and stored at $-80{ }^{\circ} \mathrm{C}$. Prior to use the sample was allowed to warm to room temperature. A solution referred to as TF/PL consisted of $30 \mathrm{pM}$ relipidated Tissue Factor (Innovin ${ }^{\circledR}$, Dade Behring, Marburg, Germany) and $24 \mu \mathrm{M}$ phospholipids in Hepesbuffered saline, prepared according to literature. ${ }^{18}$ All other reagents were used as purchased.

Hydrophilic fused silica capillaries with a polyimide coating were obtained from PolyMicro (Eerbeek, The Netherlands) with an inner diameter of $100 \mu \mathrm{m}$, outer diameter of $365 \mu \mathrm{m}$, and a length of $30 \mathrm{~cm}$ (total internal volume $2.4 \mu \mathrm{l})$.

\section{Methods}

A transparent detection window in the capillaries (typically $2-3 \mathrm{~cm}$ in length) was created by removing the coating by applying a short (typically 1-2 s) external heat source (typically a flame). The soot formed was easily removed from the capillary by a wet tissue leaving the detection window.

To construct a hydrophobic capillary, a solution of $10 \mu \mathrm{l}$ ODS-Cl (octadecyltrichlorosilane) dissolved in $1 \mathrm{ml}$ of toluene was pumped through the capillary. The solution in the capillary was left for 15-20 min. Subsequently, approximately $2 \mathrm{ml}$ of toluene $\left(1 \mathrm{ml} \mathrm{min} \mathrm{m}^{-1}\right)$ was pumped through. Finally, approximately $2 \mathrm{ml}$ of acetone was pumped through $\left(1 \mathrm{ml} \mathrm{min}{ }^{-1}\right)$ and the capillary was dried with pressurized dry air.

Conventional TGT in a 96-wells plate was carried out using the following procedure. The sample (PPP) was allowed to warm to room temperature prior to use. $80 \mu \mathrm{l}$ Sample (PPP), $20 \mu \mathrm{l}$ TF/PL solution and $20 \mu$ l FluCa were added to four wells in a 96-wells titer plate and mixed for 10 $\mathrm{s}(t=0)$. The titer plate was placed in the 96-wells plate fluorometer. The temperature was set to $25{ }^{\circ} \mathrm{C}$ and the fluorescence measurement was started (measuring every $15 \mathrm{~s}$ for 40 $\min )$.

TGT in capillaries was performed using $40 \mu \mathrm{l}$ of sample (PPP), which was premixed with $10 \mu \mathrm{l} \mathrm{FluCa}$ in order to initiate the test $(t=0)$. Subsequently, the mixed solution was brought into the capillary by applying reduced pressure and the fluorescence was measured by fluorescence microscopy.

Mixing experiments in the micromixer were performed by filling syringe I with Rhodamine B solution $(1 \mathrm{mg} / \mathrm{ml}$ in de-ionized water) and syringe II with de-ionized water. Both syringes were mounted on the syringe pumps and connected to the microchip. Pump I was programmed to deliver $50 \mu \mathrm{l}$ at a pump rate of $450 \mu \mathrm{l} \mathrm{min}^{-1}$. Pump II was programmed to

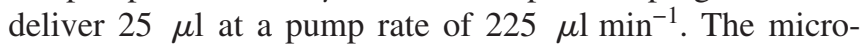
chip was positioned on the microscope (Zeiss, Axiovert 40 MAT) and the mixing experiment was initiated by starting both pumps at exactly the same time. Images were recorded with a digital camera (Carl Zeiss, Axiocam MRc5).

TGT in a micromixer was carried out by filling syringe I with sample (PPP, typically $300 \mu \mathrm{l}$ ) and syringe II with re- 

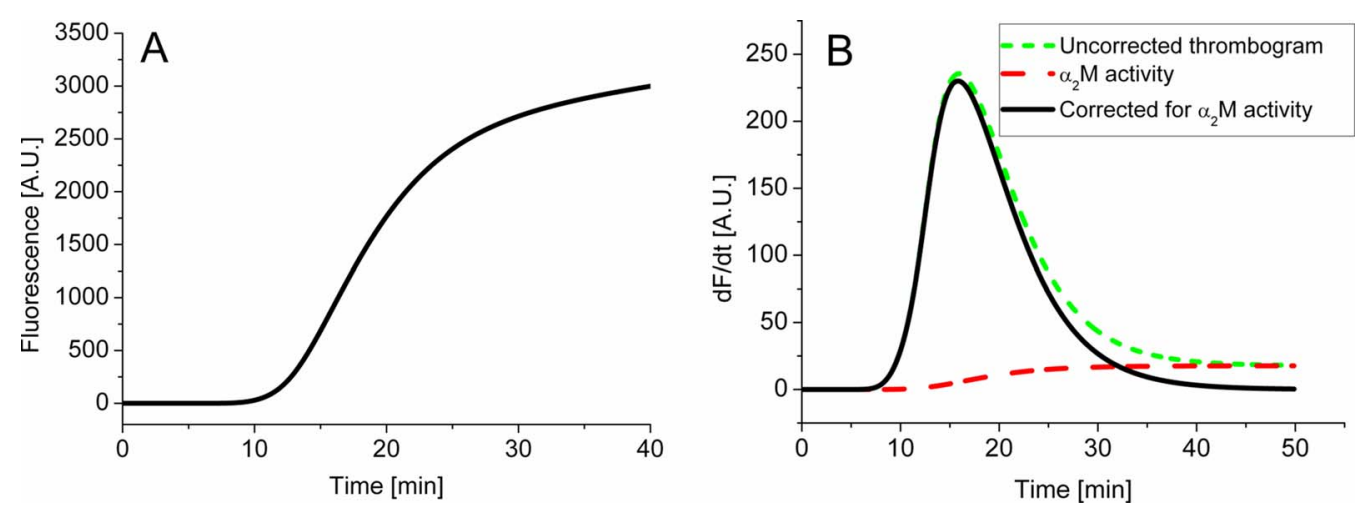

FIG. 2. (Color online) Conventional TGT at $25^{\circ} \mathrm{C}$ using the setup as depicted in Fig. 1(a) (a) Measured fluorescence in sample (PPP). (b) Thrombogram obtained after mathematical processing.

agent (typically $150 \mu \mathrm{l}$ TF/PL solution and $150 \mu \mathrm{l}$ FluCa). ${ }^{19}$ Both syringes were mounted on the syringe pumps and connected to the microreactor. Pump I was programmed to deliver $50 \mu \mathrm{l}$ at a pump rate of $450 \mu \mathrm{lmin}^{-1}$. Pump II was programmed to deliver $25 \mu \mathrm{l}$ at a pump rate of $225 \mu 1 \mathrm{~min}^{-1}$. To initiate the reaction, both pumps were started exactly at the same time, and the fluorescence in the microreactor was measured for $40 \mathrm{~min}$ by fluorescence microscopy.

\section{RESULTS AND DISCUSSION}

For reliable miniaturization of the conventional thrombin generation test several parameters such as temperature, detection limit, protein-surface interactions, and mixing behavior required profound investigation. Temperature plays an important role in many biological processes including thrombin generation. Preferably enzymatic reactions are carried out at $37{ }^{\circ} \mathrm{C}$. However, our microfluidic device was designed to function at ambient temperature. As a point of reference the conventional thrombin generation test therefore first needed to be performed in a 96-wells plate [Fig. 1(a)] at $25{ }^{\circ} \mathrm{C}$ instead of the usual $37{ }^{\circ} \mathrm{C}$.

Miniaturization of the 96-wells plate to capillaries [Fig. 1(b)] will result in sample volume reduction and efficient and reliable detection becomes an issue to address. In the conventional test the fluorophore (7-amino-4-methyl-coumarin, AMC) is liberated and measured using a standard 96-wells plate fluorometer. In the miniaturized setup [Fig. 1(b)] fluorescence microscopy was used in order to detect the fluorescent signal, and the efficiency of this method had to be determined. The use of microchannels for the TGT results in a diagnostic device with an increased surface to volume ratio when compared to the conventional test. The effect of the hydrophilicity of the channel walls therefore also had to be taken into consideration as protein-surface interactions affect coagulation behavior. Finally, in order to obtain an efficient mixing procedure of the sample and diagnostic fluid, we investigated the use of a micromixer [Fig. 1(c)].

Performing the thrombin generation test in a 96-wells plate at $25{ }^{\circ} \mathrm{C}$ resulted in a fluorescence trace as a function of time as depicted in Fig. 2(a). The thrombin activity rapidly decreases in plasma as a result of plasma inhibitors. The most important of these inhibitors, $\alpha_{2}$-macroglobulin, encap- sulates thrombin, forming a $\left(\alpha_{2}-\mathrm{M}-\mathrm{T}\right)$ complex. This $\alpha_{2}$-M-T complex switches off all biological activity of thrombin (i.e., coagulation initiation) except for the amidolytic action on small substrates. ${ }^{20}$ Consequently, there is continuous hydrolysis of fluorescent substrate (Cbz-Gly-Gly-Arg-AMC) due to the presence of the $\alpha_{2}$-M-T complex, giving rise to an increased fluorescence signal. Thus the thrombin generation curve (or thrombogram) is comprised of the fluorescent signal produced by free thrombin and the complex of thrombin with $\alpha_{2}$-macroglobulin. Mathematical processing ${ }^{21}$ was applied to convert the fluorescent signal into free thrombin activity by subtracting the $\alpha_{2} \mathrm{M}-\mathrm{T}$ activity, generating Fig. 2(b). ${ }^{22}$ The results shown in Fig. 2 demonstrate that performing the TGT at a lower temperature provided comparable thrombograms, although the time-to-peak was significantly prolonged. It could however be concluded that no intrinsic problems were encountered by performing the test at ambient temperature.

The conventional TGT consumes $80 \mu \mathrm{l}$ of sample and $40 \mu \mathrm{l}$ of reagents per test, which can easily be reduced upon usage of capillaries or other microfluidic systems. In the first miniaturized experiments, the sample and reagents were premixed outside a microchannel, after which the mixture was introduced into a capillary by reduced pressure and the thrombin generation was measured [schematically depicted in Fig. 1(b)]. Initially, we tested the TGT in fused silica capillaries with an inner diameter of $100 \mu \mathrm{m}$ (total internal volume $2.4 \mu \mathrm{l}$ ). To investigate the effect of the polarity of the surface, both hydrophobic and hydrophilic capillaries were examined at $25^{\circ} \mathrm{C}$ (Fig. 3).

The hydrophobicity has a significant effect on the time to peak, as shown in Fig. 3. Using a hydrophobic channel resulted in a delay of the time to peak of approximately $13 \mathrm{~min}$ in comparison with the hydrophilic channel (17.8 and 4.7 min., respectively). This could be explained by the enhanced binding of proteins to the hydrophilic surface of the capillary hence increasing the coagulation activity via the intrinsic pathway (i.e., coagulation in a blood vessel initiated by the activation by certain negatively charged surfaces ${ }^{23}$ ). Comparing the results of both capillaries with the established 96wells plate results, the hydrophobic capillary yielded the more similar outcome, with no significant difference in the time-to-peak parameter (17.8 and $15.8 \mathrm{~min}$, respectively). 


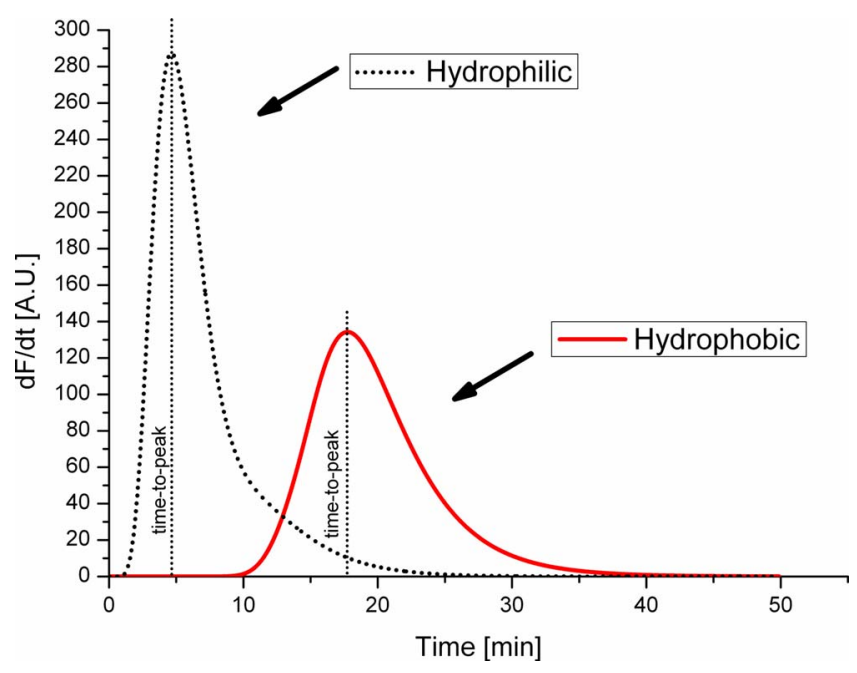

FIG. 3. (Color online) Thrombograms of both hydrophilic and hydrophobic capillaries. For clarity reasons only the corrected TG curves are depicted.

To demonstrate that thrombin generation could also be measured in a microchip [using a commercially available micromixer, as depicted in Fig. 1(c)], we initially applied the premixing method as described for the capillaries prior to injection into the microchip. The fluorescence signal was monitored during $40 \mathrm{~min}$ and after mathematical processing, ${ }^{21}$ the thrombogram was generated (Fig. 4).

The obtained thrombogram (Fig. 4) demonstrates that the thrombin generation can be monitored in the micromixer. Remarkably, the time to peak (13.2 min) was situated in between the times found for the hydrophobic and hydrophilic fused silica capillaries while the material of the microchip is hydrophilic (untreated borosilicate glass). The increased time to peak might be explained by the slightly different composition of the channel material and the lower surface-tovolume ratio of the microchip compared to the capillary which could decrease the intrinsic coagulation activation.

Encouraged by these results, we investigated the possibility to eliminate the premixing step by applying an excellent micromixer chip. ${ }^{24,25}$ To evaluate the mixing behavior of this micromixer, two aqueous solutions (one containing the fluorescent dye Rhodamine $\mathrm{B}$, the other de-ionized water) were pumped at a controlled rate through the micromixer.

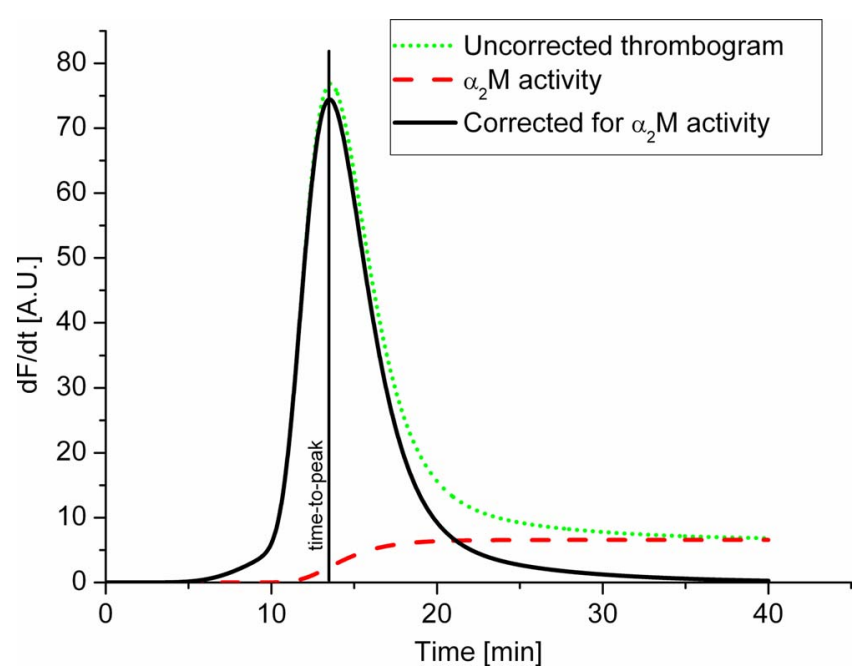

FIG. 4. (Color online) Thrombogram recorded in the micromixer of sample and reagents premixed prior to injection.

Figure 5 shows that after four mixing units mixing was complete. By replacing the aqueous solution with a BSA buffer solution (closer related to physiological conditions), similar mixing behavior was observed. ${ }^{21}$

Having established that the mixing in a micromixer occurs rapidly, we proceeded by performing the TGT in this specific micromixer. The premixing step was eliminated by introducing the sample and reagent via separate syringes into the microchip, as depicted in Fig. 1(c). The pump rate for the two solutions was set at a ratio of 2:1 (sample:reagents). After loading the microchip with the appropriate amount of sample and reagent (approximately $4 \mu \mathrm{l}$ ) thrombin generation was monitored during a period of $40 \mathrm{~min}$. To our satisfaction we obtained the thrombogram, as depicted in Fig. 6.

Comparing the on-chip mixing procedure with the premix method, modest variations in time to peak and peak height were observed. The distribution is slightly broadened indicating a delayed and prolonged thrombin generation. This can possibly be explained by the low accessibility of $\mathrm{TF}$, which could be improved by changing the mixing ratio. ${ }^{26}$ Based on these results it can thus be concluded that the TGT can be performed in a micromixer with efficient on-chip mixing, lower sample volumes, and excellent detection.

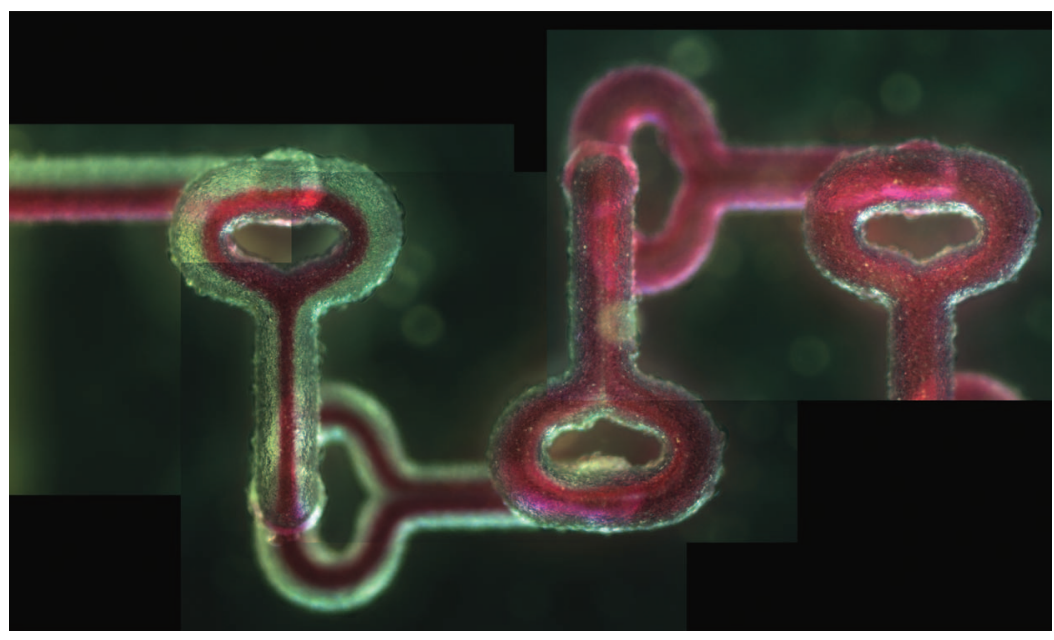

FIG. 5. (Color) Combined images of five consecutive mixing units of a mixing experiment in the microreactor. Flow direction is from left to right. One of the aqueous phases is colored with a dye (Rhodamine B) to visualize the mixing behavior in the micromixer. 


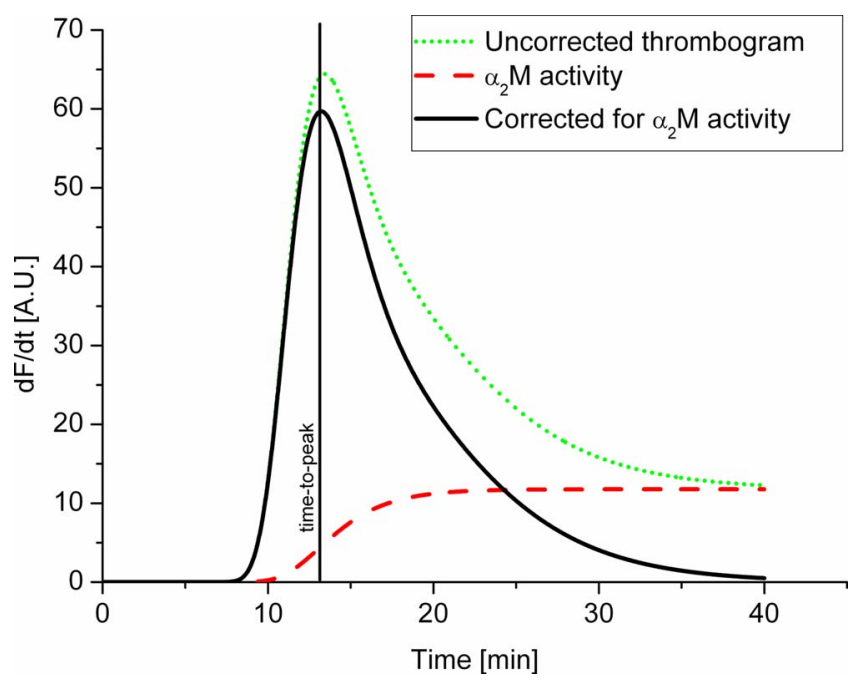

FIG. 6. (Color online) Thrombogram of the TGT in the micromixer with on-chip mixing of the sample and reagents.

\section{CONCLUDING REMARKS}

The focus of our research was to investigate the miniaturization of the conventional thrombin generation test by applying microfluidic devices. We demonstrated that thrombin generation can be measured in different microfluidic devices with excellent detection. The use of microfluidics resulted in 30-fold sample volume reduction. The influence of protein-surface interaction was demonstrated using both hydrophilic and hydrophobic capillaries resulting in a large shift in time to peak possibly as a result of contact activation of the intrinsic coagulation pathway by the glass surface. The use of a micromixer enabled us to perform efficient on-chip mixing, which resulted in the successful measurement of a thrombin generation curve. With this work we demonstrated that microreactor technology is suitable for miniaturization of the conventional thrombin generation test. As a next step validation of this test by calibrating the fluorescent signal and performing the TGT in a hydrophobic micromixer will be examined.

\section{ACKNOWLEDGMENTS}

This research supported with financial aid from EUREGIO Rhine-Waal (Interreg IIIA) and the Netherlands Technology Foundation (STW). The authors thank Dr. E. J. Lous (NXP Semiconductors, Nijmegen, The Netherlands) and Dr. W. L. van Heerde (Thrombosis Hemostasis Research Unit, UMC St Radboud, Nijmegen, The Netherlands) for useful discussions. K.K. and S.S.v.B. contributed equally to this work.

\section{ABBREVIATIONS}

\author{
Cbz carbobenzyloxy \\ Gly glycine \\ Arg arginine \\ AMC amino-4-methylcoumarin (AMC)
}

${ }^{1}$ World Health Organization Factsheet 2002 online (http://www.who.int/ mediacentre/factsheets/fs310/en/index.html).

${ }^{2}$ P. Yager, T. Edwards, E. Fu, K. Helton, K. Nelson, M. R. Tam, and B. H. Weigl, Nature (London) 442, 412 (2006).

${ }^{3}$ H. C. Hemker, P. L. A. Giesen, M. Ramjee, R. Wagenvoord, and S. Béguin, Thromb. Haemostasis 83, 589 (2000).

${ }^{4}$ V. Chantarangkul, M. Clerici, C. Bressi, P. L. A. Giesen, and A. Tripodi, Haematologica 88, 547 (2003).

${ }^{5}$ H. C. Hemker, P. Giesen, R. Al Dieri, V. Regnault, E. de Smedt, R. Wagenvoord, T. Lecompte, and S. Béguin, Pathophysiol. Haemost. Thromb. 33, 4 (2003).

${ }^{6}$ P. von Lode, Clin. Biochem. 38, 591 (2005).

${ }^{7}$ C. P. Price, BMJ 322, 1285 (2001).

${ }^{8}$ A. Manz, N. Graber, and H. M. Widmer, Sens. Actuators B 1, 244 (1990).

${ }^{9}$ S. Haeberle and R. Zengerle, Lab Chip 7, 1094 (2007).

${ }^{10}$ C. Haber, Lab Chip 6, 1118 (2006).

${ }^{11}$ R. Irawan, C. Tjin Swee, P. Yager, and D. Zhang, Biomed. Microdevices 7, 205 (2005).

${ }^{12}$ C. T. Schembri, T. L. Burd, A. R. Kopfsill, L. R. Shea, and B. Braynin, J. Autom. Chem. 17, 99 (1995).

${ }^{13}$ S. Haeberle, T. Brenner, R. Zengerle, and J. Ducrée, Lab Chip 6, 776 (2006).

${ }^{14}$ S. Yang, A. Undar, and J. D. Zahn, ASAIO J. 51, 585 (2005); Lab Chip 6, 871 (2006).

${ }^{15}$ X. Yang, A. Hibara, K. Sato, M. Tokeshi, K. Morishima, Y. Kikutani, H. Kimura, T. Kitamori, Special Publication-Royal Society of Chemistry, 296, Proceedings of Micro Total Analysis Systems, Volume 1, p. 120 (2004).

${ }^{16}$ H. Song, H.-W. Li, M. S. Munson, T. G. Van Ha, and R. F. Ismagilov, Anal. Chem. 78, 4839 (2006).

${ }^{17}$ H. K. Trieu, M. Bollerott, M. Kemmerling, R. Hilderbrand, J. C. M. van Hest, F. P. J. T. Rutjes, K. Koch, P. J. Nieuwland, T. A. van Beek, E. J. R. Südholter, R. M. Boom, and A. E. M. Janssen, WO Patent No. 2007016931 (15 February 2007).

${ }^{18}$ S. Wielders, M. Mukherjee, J. Michiels, D. T. S. Rijkers, J. P. Cambus, R. W. Knebel, V. Kakkar, H. C. Hemker, and S. Béguin, Thromb. Haemostasis 77, 629 (1997).

${ }^{19}$ The total volume of plasma and reagents needed to conduct the TGT in the micromixer was rather large due to the fact that relatively large syringes and tubing were used. This can easily be downscaled to very small volumes and will be subject of investigation in future research.

${ }^{20}$ D. T. S. Rijkers, S. J. H. Wielders, S. Béguin, and H. C. Hemker, Thromb. Res. 89, 161 (1998).

${ }^{21}$ See EPAPS Document No. E-JAPIAU-105-044992 for an example for the mathematical processing of the data. For more information on EPAPS, see http://www.aip.org/pubservs/epaps.html.

${ }^{22}$ T. Baglin, Br. J. Haematol. 130, 653 (2005).

${ }^{23}$ D. Gailani and T. Renné, J. Thromb. Haemost. 5, 1106 (2007).

${ }^{24}$ N. Blow, Nat. Methods 4, 665 (2007).

${ }^{25}$ M. Nguyen, Z. Wu, J. Micromech. Microeng. 15, R1 (2004).

${ }^{26}$ K. Lo and S. L. Diamond, Thromb. Haemostasis 92, 874 (2004). 